\title{
Traumatic brain injury, the hidden pandemic: A focused response to family and patient experiences and needs
}

\author{
J Webster, ${ }^{1}$ MA (Sociology, Philosophy); A Taylor, ${ }^{2}$ MB BCh, FCS (Neurosurgery), MMed; \\ R Balchin, ${ }^{3}$ BSocSci, MA (Neuropsychology), PhD (Neuropsychology) \\ ${ }^{1}$ ComaCARE Trust, Groote Schuur Hospital, Cape Town, South Africa \\ ${ }^{2}$ Division of Neurosurgery, Faculty of Health Sciences, University of Cape Town and Groote Schuur Hospital, Cape Town, South Africa \\ ${ }^{3}$ Department of Psychology, Faculty of Humanities, University of Cape Town, South Africa
}

Corresponding author: A Taylor (allan.taylor@uct.ac.za)

\begin{abstract}
Introduction. Traumatic brain injury (TBI) has many potential cognitive, behavioural and psychological consequences, and contributes significantly to the national burden of disease and to ongoing violent behaviour. Few resources are available for the rehabilitation of patients with TBI in South Africa, and access to rehabilitation facilities in the public sector is limited. Consequently, it is the families impacted on by TBI that ultimately carry the care and rehabilitation burden once survivors are discharged from hospital. Families are generally ill equipped to cope with the complex and potentially long-term disabilities that accompany brain injury.

Methods. Reviewing interviews with 175 family members and 354 patients recovering from TBI helped identify the key challenges that the survivors of TBI and their families face.

Results. Nine problem areas were identified that formed the basis for development of a discharge resource, the S-Plan, which serves to inform patients and carers and provide practical solutions for the problems they face.

Conclusion. The experiences of TBI survivors and their family members served to inform the development of simple, integrated coping strategies, namely two S-Plan tools, one for survivors and their families/caregivers and the other for care workers, in conjunction with counselling and support group processes. The S-Plan constitutes a discharge resource to inform patients and carers and provide practical solutions for the problems they face in caring for family members who have suffered TBI.

S Afr Med J 2015;105(3):195-198. DOI:10.7196/SAMJ.9014
\end{abstract}

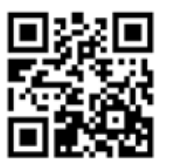

\section{The context of trauma}

In South Africa (SA), the high prevalence of violence contributes to the national burden of disease. ${ }^{[1]}$ In the Western Cape Province in 2009, $7 \%$ of deaths ( $n=3$ 217) were caused by intentional injuries, which also accounted for $9.7 \%$ of years of life lost (YLL) - a total of 79653 YLL. ${ }^{[2]}$

\section{Traumatic brain injury (TBI) upstream factors and injury profile}

In 2009, an internal audit conducted at Groote Schuur Hospital (GSH), Cape Town, Western Cape, revealed that of the total of 10046 trauma patients admitted that year, approximately $24 \%$ were classified as head-injury patients, with 654 having a moderate to severe TBI. The direct cost of these patients to the tertiary service in 2009 was R17 448756 (T King, Coma Care Trust audit, 2010 - unpublished). This audit also identified that $82 \%$ of assault-related head trauma was experienced by young black and coloured men, and that the average length of stay in hospital for a patient receiving neurosurgical intervention was 10.2 days. This brief slice of the patient's life in the acute-care setting is usually preceded by an upstream reality involving poverty, unemployment, poor community infrastructure, high rates of alcohol consumption and a weakened family unit.

\section{Downstream impact of TBI}

TBI can require a long and arduous recovery process. Many survivors are left with permanent physical, emotional and cognitive disabilities. TBIs that are caused by interpersonal violence are inflicted largely by right-handed perpetrators, and typically result in frontal lobe and/or left temporal lobe injuries. The neurocognitive and behavioural sequelae of frontal lobe injuries are severe and include poor judgement, impaired problem-solving ability and loss of the ability to think abstractly, poor organisational skills, loss of inhibition and impulsive behaviour, aggression, personality changes, depression, anxiety and reduced social skills. ${ }^{[3-5]}$ Left temporal lobe injuries can result in communication difficulties due to disorders of language (receptive aphasia), including loss of the ability to comprehend speech. ${ }^{[3-6]}$ These deficits have a disabling effect on survivors' ability to cope with activities of daily living and with constructive engagement within their families and communities. Poor impulse control and weak social skills result in dangerous situations for survivors and for those around them.

The link between TBI and criminal behaviour is internationally well evidenced. In a Finnish study, adolescent TBI survivors were found to have committed crimes significantly more often than adolescents without a TBI $\left(53.8 \%\right.$ v. $14.7 \%$, respectively) ${ }^{[7]}$ In addition, the prevalence of both violent crimes ( $42.9 \%$ v. $9.1 \%$ ), and non-violent crimes $(29.4 \%$ v. $6.8 \%)$ was also higher in the TBI group. Similarly, in a study of 186 young offenders in the UK, the frequency of self-reported TBI was associated with more convictions; three or more self-reported TBIs were associated with greater violence in the offences that were committed. ${ }^{[8]}$ The rehabilitation of TBI survivors is therefore not only a health issue for survivors and their families, but also a critical violence prevention strategy for SA.

\section{The current rehabilitation response}

In SA, there is limited access to rehabilitation facilities in the public sector. The consequence for TBI survivors is that very few receive adequate rehabilitation services; only 16 of the 654 survivors in the 2009 GSH audit were admitted to a public rehabilitation facility. Over a 5-year period (2008 - 2012), TBI survivors made up less 
than 9\% of the Western Cape Rehabilitation Centre (WCRC)'s intake (Dr Jenny Hendry, personal communication, 2014). The WCRC is a 240 -bed rehabilitation centre and the only dedicated rehabilitation service available to public patients. In addition, a TBI may include an initial period of post-traumatic amnesia and usually involves a slow recovery period, yet candidates for rehabilitation centres are expected to behave appropriately and recover in a 6 12 -week period. This means that few survivors receive the necessary rehabilitation support, and the majority are instead discharged to unprepared families who typically become their main support structure. This ultimately negatively affects caregiver wellness and family resilience. ${ }^{[9,10]}$ Outpatient rehabilitation is also very limited, and most patients receive no more than 30 minutes of physiotherapy or occupational therapy per week at their community healthcare centre.

\section{Addressing the need}

The ComaCARE Trust is a non-profit organisation based at GSH. ComaCARE has been addressing the needs of survivors and families since 2005 by providing a psychosocial service that employs community caregivers in the acute-care setting. In 2012, recognising that TBI survivors are usually discharged into the care of an unprepared family unit, ComaCARE developed a brain injury prevention and family support service in Khayelitsha, one of the Western Cape's hot spots for interpersonal violence as identified through injury mortality surveillance data. ${ }^{[2]}$ This HeadsUP! Hub provides supportive intervention for families that involves counselling by social workers and training to know what to do if their relative displays any of the sequelae of TBI (e.g. disruptive behaviour at school or in the workplace, aggression, memory problems, anxiety, depression and sleep disturbances).

\section{Objectives}

To faciliate the provision of relevant and sustainable TBI services, this study had two primary objectives: (i) to examine in a systematic way the key challenges that survivors of brain injury and their family members face; and (ii) to create tools as guides in helping families and care workers to manage and cope with the consequences of brain injury in under-resourced settings.

\section{Methods}

This was an applied, exploratory study. The approach to data collection was qualitative, as the interview data collected were analysed thematically to extract and highlight the topics that emerged. Participants (families of TBI survivors and the survivors themselves) were identified using purposive sampling. The overall study design had two distinct phases: (i) the process of identifying the key problems faced by survivors of TBI and their families through interviews gathered from various sources (see below); and (ii) the process of creating the content for tools to serve as guides in assisting families and care workers to manage and cope with the consequences of brain injury. This second phase was dependent on the data collected during phase one, which served to inform the content and structure of the tools created.

The experiences of 175 family members and 354 survivors were recorded. These interview data were collected between 2008 and 2013 from: (i) the acute-care hospital support service offered by ComaCARE at GSH; (ii) intake interviews for the HeadsUP! survivor care programme; (iii) bimonthly outpatient support service clinics linked to the Division of Neurosurgery at GSH; and (iv) HeadsUP! support groups. In both the individual and the group sessions, survivors and family members were interviewed separately, thereby enabling each group to express themselves freely. Feedback from all sources was recorded in written notes, which were subsequently analysed thematically to extract and highlight the common topics that emerged.

\section{Results}

\section{Phase 1: Family and survivor feedback}

Several critical factors emerged from the data collection process to aid the provision of better services for TBI survivors and their families.

\section{Lack of accessible information}

In general, it was evident that there was initially a communication problem between family members and medical professionals in the acute-care setting. Family members felt that they had not been adequately informed by medical professionals about TBI and its potential consequences. When they did receive feedback, it was often too complex to be understood, families lacking a frame of reference to be able to contextualise any knowledge received. Families related that in their traumatised state, they were unable to absorb spoken information and then convey it to other family members. Family feedback also identified the clear lack of information regarding the longer-term requirements of recovery and possible strategies to cope with cognitive impairments, personality changes and behavioural issues.

\section{Content of required information}

The key themes that emerged from families, and the areas where information is desperately needed, included coping with personality changes, how to manage aggressive behaviour, issues regarding memory, issues regarding motivation, sleep disturbances, language and communication problems, sexual disinhibition and/or disinterest, depression and safety concerns. Family members reported that their own mental health was adversely affected as a result of the stress of having to look after survivors; depression, fatigue, utter frustration and burnout were commonly reported.

The issues most commonly reported by the TBI survivors (who often revealed a lack of insight into their neurocognitive and behavioural problems) were the need for a social life and meaningful activity, sensitivity to noise, difficulty sleeping, depression, anxiety and headaches. Some survivors also reported that family members no longer had the time or patience to engage and listen to them, and consequently failed to understand their problems, their needs and their frustrations. The issues of depression, aggressive behaviour, headaches and anxiety were probed through further questioning in order to establish whether there were any triggers that preceded these experiences.

\section{Type of information and support}

Families stated that at the acute stage of injury, verbal information alone was not adequate, given that they needed time to review information and to share it with family members. On the survivor's return home, families reported that their information needs changed, as survivors' behavioural issues only became apparent as new challenges presented over time. Families therefore needed written information that could be consulted at different stages of the recovery process, and that could be used to help the survivor to take part in his/her own rehabilitation process wherever possible. In addition, both families and survivors expressed the need for access to advisors and counsellors, and wanted to counter their feelings of isolation in fora where people could understand and relate to their problems. 
Finding practical solutions

Some of the more experienced family members interviewed described their coping strategies to avoid and manage the most distressing survivor behaviours, noting that, as with parenting a child, survivors needed to have a balanced and structured day to ensure a sense of wellbeing and safety, and that they needed regular sleep, a healthy diet, stimulation and friendship. The problems experienced, and the strategies that families employed, were then analysed and compiled under nine central topics/themes based on words starting with 'S' (Fig. 1). This decision to incorporate the key topics identified under headings that all began with the same letter (in English) was made in order to make them easier to remember and to provide a framework for the varied information from the often very different sources available. English was chosen as the language of written communication, as many of the participants refused to accept written information in isiXhosa, despite its being their home language.

\section{Phase 2: The development of the S-Plan process \\ Survivors and families identifying challenges}

Two sets of posters were designed (one with images of adults and the other of children) in order to allow for both survivors and family members to have each of the elements of the poster explained to them by a care worker who speaks English and also their home language. In their use, the care worker explains the behaviours and challenges that families may experience on the road to recovery, or that trigger, or are triggered by, difficult emotions or symptoms. Family members and survivors are then asked whether they are currently facing difficulties in any of these areas. If one of the ' $S$ issues' is identified through this process, the care worker will interview the family member and survivor separately using the questionnaires pertaining to the relevant component of the S-Plan. Next, in discussion with both parties, the care worker will support them in choosing one or two areas to focus on in the coming weeks.

\section{Support with written material, counselling} and engagement in support groups

After an agreed issue to work on has been identified, an S-Plan guide booklet is given to the family member and survivor. This guide contains an introduction and also covers all nine topics, with each of the sections including information under the following headings: 'What is the problem?',

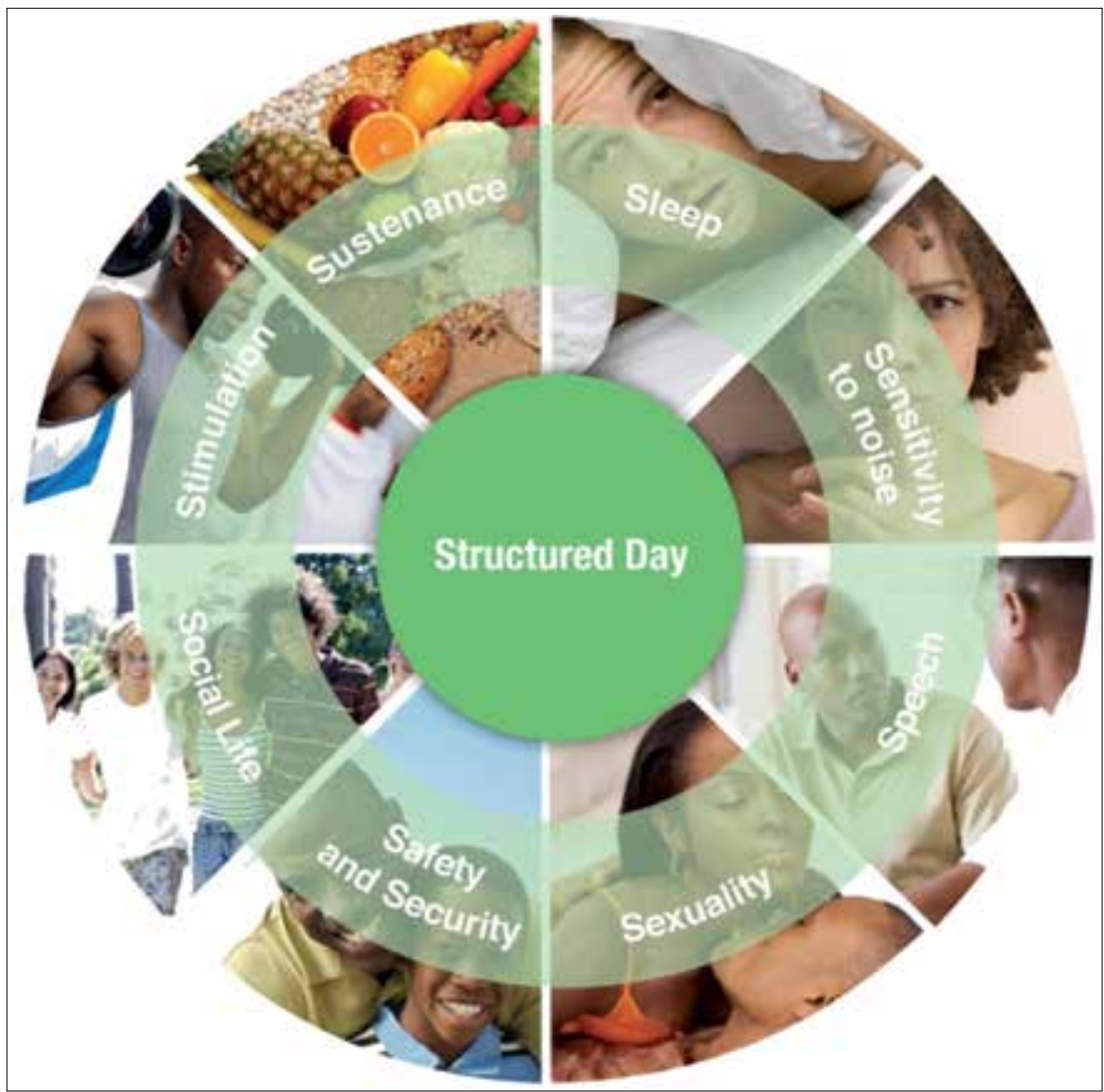

Fig. 1. The S-Plan.

'When it is likely to happen', 'What it looks like, 'What can make things worse?', 'How does this make life difficult for survivors?', 'How it might make you feel as a caregiver', 'What you can do to help', and 'Who can help further?'. These guides are currently used with family units, and at the three HeadsUP! support groups that were established in 2012. Posters for teaching and training purposes have also been designed to accompany these $\mathrm{S}$-Plan guides. The posters include spaces for the nine words starting with ' $\mathrm{S}$ ' to be translated when necessary. In the future, the guides will be translated into isiXhosa/ English and Afrikaans/English versions.

\section{Discussion}

SA faces many healthcare challenges, including a high burden of physical trauma with few rehabilitation services. Funding limitations and the demand for additional acute-care services mean that it is unlikely that new rehabilitation services will be added in the near future, and patients will continue to be discharged home from acutecare settings. While this study focused on TBI patients treated in Cape Town, it is likely that TBI patients and their families throughout SA face the same, or worse, difficulties. Inpatient admission times are typically short (10 days on average) and acute rehabilitation during this time is limited, with many families having to take on the burden of looking after a person with impairments such as hemiparesis, dysphasia, confusion and aggression. No local studies have surveyed outcomes for TBI survivors who are discharged early into a setting with limited outpatient rehabilitation, although the outcomes of stroke patients discharged in similar circumstances in Cape Town have been examined. One of the findings from a study examining survival, disability and functional outcomes of stroke patients following discharge was that patients requiring nasogastric feeding did particularly poorly at home, indicating that their caregivers were not equipped to deal with the complexity of the care required. ${ }^{[11]}$

It is evident that TBI is a huge problem globally, and that families ultimately bear the burden of care provision, yet there is little available literature from underresourced communities regarding the needs of families of survivors and the problems they face. ${ }^{[12]}$ Studies (mainly in developed countries) that have looked at families' needs (both met and unmet) and the problems they face in relation to TBI have identified high levels of stress, lack of education regarding TBI, lack of information on how to prepare for the 
future, behavioural problems and problems at school on the part of the TBI survivor, and survivors' loss of interest in activities, anxiety, memory problems and aggressive temperament. ${ }^{[12-16]}$ Commonly reported needs, which like the reported problems can change over time, include the need for social/instrumental care, emotional care and healthcare; the need for professional and financial support and information; the need for access to relevant information regarding TBI and its consequences; and the need to be actively involved with the care of the TBI survivor. ${ }^{[12-15]}$

Successful TBI support will not only benefit survivors and their family units, but can also contribute towards a reduction in future violent acts perpetrated by TBI survivors. In SA, as in many other parts of the world, inadequate information and support, along with the lack of appropriate rehabilitation facilities, places a massive care burden on unprepared family members and community care workers. The participants in this study identified a multitude of complex daily challenges when survivors returned to their homes and communities. These challenges cover a wide spectrum, ranging from survivors' behavioural and memory problems to depression and sleep disturbances. Caregivers' own mental health is negatively affected, with depression, fatigue, stress and frustration all reported. Consequently, the ability of families to cope is often compromised, adversely affecting TBI recovery.

The S-Plan tools (the family guide, posters and questionnaires) have been created to compensate for the lack of resources that are necessary to respond to the hidden pandemic that is TBI. The provision of these tools aims to empower families, survivors and healthcare professionals by offering a structured and interactive approach to identifying and then addressing the specific challenges that each TBI survivor and their family members face. The S-Plan has been developed to educate families and survivors about the need for a balanced, structured day, and will help families to reflect on the triggers of disruptive behaviour and to plan the survivor's rehabilitation process. It also raises taboo subjects such as sexuality, substance abuse and security, and enables conversation about concerns and fears around such subjects. The questionnaires have been designed with specific emphasis on: (i) helping to identify the perceptions of survivors and families about their behaviours; (ii) revealing where there is a disabling lack of insight, sometimes from both parties; and (iii) helping survivors and families to decide together on priority areas for goal setting.

The ComaCARE Trust acknowledges families as the experts in this process, and hopes to strengthen their advocacy role in exposing the hidden epidemic of TBI and the dangers inherent in failing to provide adequate rehabilitation services that can impact favourably on family wellness and violence prevention. Further research will be conducted in communities in the Western Cape in order to systematically investigate the efficacy of the new S-Plan tools. This will involve an iterative research process, testing separate elements of the S-Plan tools based on families' and care workers' firsthand experience of their effectiveness, with the aim of improving them.

\section{Conclusion}

The experiences of TBI survivors and their family members have served to inform the development of simple, integrated coping strategies (S-Plan tools in conjunction with counselling and support group processes). One S-Plan tool is for survivors and their families/ caregivers, and the other is for care workers. For the next phase of this research, these integrated tools will be piloted from 2015 onwards as part of a longitudinal research project to be conducted in underresourced communities in Cape Town.

Acknowledgements. JW thanks the Nussbaum Foundation, the Norwegian Centre for Human Rights and the Western Cape Department of Social Development for their support of this research. RB sincerely thanks the Claude Leon Foundation for their funding and support of this research.

\section{References}

1. Matzopoulos R, Bowman B, Mathews S, Myers J. Applying upstream interventions for interpersonal violence prevention: An uphill struggle in low- to middle- income contexts. Health Policy 2010;97(1):62-70. [http://dx.doi.org/10.1016/j.healthpol.2010.03.003]

2. Groenewald P, Bradshaw D, Msemburi W, et al. Western Cape Mortality Profile 2009. Cape Town: South African Medical Research Council, 2012. http://www.mrc.ac.za/bod/ We

3. Walsh K, Darby EJ. Neuropsychology: A Clinical Approach. 4th ed. Edinburgh: Churchill Livingstone, 1999.

4. McDonald BC, Flashman LA, Saykin AJ. Executive dysfunction following traumatic brain injury: Neural substrates and treatment strategies. NeuroRehabilitation 2002;17(4):333-344

5. Kolb B, Whishaw IQ. Fundamentals of Human Neuropsychology. 5th ed. Alberta: Worth Publishers, 2003

6. Stuss DT. Traumatic brain injury: Relation to executive dysfunction and the frontal lobes. Curr Opin Neurol 2011;24(6):584-589. [http://dx.doi.org/10.1097/WCO.0b013e32834c7eb9]

7. Luukkainen S, Riala K, Laukkanen M, Hakko H, Räsänen P. Research Reports - Association of traumatic brain injury with criminality in adolescent psychiatric inpatients from Northern Finland. Psychiatry Res 2012;200(2-3):767-772. [http://dx.doi.org/10.1016/j.psychres.2012.04.018]

8. Williams WH, Cordan G, Mewse AJ, Tonks J, Burgess CN. Self-reported traumatic brain injury in Williams $\mathrm{WH}$, Cordan $\mathrm{G}$, Mewse AJ, Tonks J, Burgess $\mathrm{CN}$. Self-reported traumatic brain injury in
male young offenders: A risk factor for re-offending, poor mental health and violence? Neuropsychol male young offenders: A risk factor for re-offending, poor mental health and vi

9. Chesnut RM, Carney N, Maynard H, et al. Evidence Report on Rehabilitation of Persons with Traumatic Brain Injury. Rockville, Md: Agency for Health Care Policy and Research, 1998. http://www. ncbi.nlm.nih.gov/books/NBK32897/ (accessed 9 October 2014)

10. Carney N, du Coudray H, Davis-O'Reilly C, et al. Rehabilitation for Traumatic Brain Injury in Children and Adolescents. Evidence Report No. 2, Supplement. Rockville, Md: Agency for Health Care Policy and Research, 1999. http://www.ncbi.nlm.nih.gov/books/NBK32918/ (accessed 9 October 2014).

1. De Villiers L, Badri M, Ferreira M, Bryer A. Stroke outcomes in a socio-economically disadvantaged urban community. S Afr Med J 2011;101(5):345-348.

12. Hassan STS, Khaw WF, Rosna AR, Husna J. Traumatic brain injury: Caregivers' problems and needs. J Nepal Med Assoc 2011;51(181):53-55

13. Rotondi AJ, Sinkule J, Balzer K, Harris J, Moldovan R. A qualitative needs assessment of persons who have experienced traumatic brain injury and their primary family caregivers. J Head Trauma Rehabil 2007:22(1):14-25. [http://dx.doi.org/10.1097/00001199-200701000-00002]

14. Arango-Lasprilla JC, Quijano MC, Aponte M, et al. Family needs in caregivers of individuals with traumatic brain injury from Colombia, South America. Brain Inj 2010;24(7-8):1017-1026. [http:// dx.doi.org/10.3109/02699052.2010.490516]

15. Hawley CA. Reported problems and their resolution following mild, moderate and severe traumatic brain injury amongst children and adolescents in the UK. Brain Inj 2003;17(2):105-129. [http://dx.doi. org/10.1080/0269905021000010131]

16. Hall KM, Karzmark P, Stevens M, Englander J, O’Hare P, Wright J. Family stressors in traumatic brain injury: A two-year follow-up. Arch Phys Med Rehabil 1994;75(8):876-884. [http://dx.doi. org/10.1016/0003-9993(94)90112-0]

Accepted 20 October 2014 